\title{
Innovative radiation oncology Together - Precise, Personalized, Human
}

\author{
Vision 2030 for radiotherapy \& radiation oncology in Germany
}

\author{
David Krug ${ }^{1} \cdot$ Markus Hecht $^{2} \cdot$ Nadja Ebert $^{3} \cdot$ Matthias Mäurer $^{4}$ - Daniel F. Fleischmann ${ }^{5}$ Emmanouil Fokas $^{6}$. \\ Christoph Straube ${ }^{7,8}$. Nils Henrik Nicolay ${ }^{9}$ Juliane Hörner-Rieber ${ }^{10}$ • Daniela Schmitt ${ }^{11}$ • Cläre von Neubeck ${ }^{12}$. \\ Constantinos Zamboglou ${ }^{9}$ Elena Sperk ${ }^{13}$. David Kaul ${ }^{14}$. Julia Hess ${ }^{15}$. Stefanie Corradini ${ }^{5}$ Clemens Seidel $^{16}$. \\ Cihan Gani ${ }^{17}$. Christian Baues ${ }^{18}$ • Benjamin Frey ${ }^{2}$ Oliver Blanck ${ }^{1}$ Tobias Gauer ${ }^{19}$ - Maximilian Niyazi ${ }^{5}$ iD
}

Received: 4 June 2021 / Accepted: 9 August 2021 / Published online: 13 September 2021

(C) The Author(s) 2021, corrected publication 2021

\begin{abstract}
Purpose Scientific and clinical achievements in radiation, medical, and surgical oncology are changing the landscape of interdisciplinary oncology. The German Society for Radiation Oncology (DEGRO) working group of young clinicians and scientists (yDEGRO) and the DEGRO representation of associate and full professors (AKRO) are aware of the essential role of radiation oncology in multidisciplinary treatment approaches. Together, yDEGRO and AKRO endorsed developing a German radiotherapy \& radiation oncology vision 2030 to address future challenges in patient care, research, and education. The vision 2030 aims to identify priorities and goals for the next decade in the field of radiation oncology.
\end{abstract}

Original German phrase: Innovative Radioonkologie im TeamPräzise, Personalisiert, Menschlich.

Shared first authorship: D. Krug and M. Hecht; shared senior authorship: T. Gauer and M. Niyazi.

List of all participants of the yDEGRO/AKRO Retreat November 15, 2019 (in alphabetical order) Irenäus A. Adamietz, Christoph Arnold, Christian Baues, René Baumann, Claus Belka, Oliver Blanck, Kerstin Borgmann, Volker Budach, Wilfried Budach, Stephanie E. Combs, Nils Cordes, Stefanie Corradini, Christian T. Dietzel, Sophie Dobiasch, Yvonne Dzierma, Nadja Ebert, Michael J. Eble, Franziska Eckert, Astrid Faller, Julius Fischer, Thomas Fischer, Daniel F. Fleischmann, Emmanouil Fokas, Benjamin Frey, Ute Ganswindt, Tobias Gauer, Hans Geinitz, Frank A. Giordano, Christian Graeff, Burkhard Greve, Anca-Ligia Grosu, Maja Guberina, Nika Guberina, Daniel Habermehl, Franziska D. Hauth, Uwe Haverkamp, Christoph Henkenberens, Julia Hess, Juliane Hörner-Rieber, Stefan Joos, Lukas Käsmann, David Kaul, Diana Klein, Mechthild Krause, David Krug, Kirsten Lauber, Anastassia Löser, Matthias Mäurer, Nils H. Nicolay, Maximilian Niyazi, Katia Parodi, Cordula Petersen, Florian Putz, Felicitas Rapp, Thorsten Rieckmann, Daniela Schmitt, Annemarie Schröder, Andrea Schwahofer, Clemens Seidel, Elena Sperk, Sarah Stefanowicz, Christoph Straube, Martin Stuschke, Kristian Unger, Vincenzo Valentini, Cläre von Neubeck, Andrea Wittig, Marcus Zimmermann

Extended author information available on the last page of the article 
Methods The vision development comprised three phases. During the first phase, areas of interest, objectives, and the process of vision development were defined jointly by the yDEGRO, AKRO, and the DEGRO board. In the second phase, a one-day strategy retreat was held to develop AKRO and yDEGRO representatives' final vision from medicine, biology, and physics. The third phase was dedicated to vision interpretation and program development by yDEGRO representatives. Results The strategy retreat's development process resulted in conception of the final vision "Innovative radiation oncology Together - Precise, Personalized, Human." The first term "Innovative radiation oncology" comprises the promotion of preclinical research and clinical trials and highlights the development of a national committee for strategic development in radiation oncology research. The term "together" underpins collaborations within radiation oncology departments as well as with other partners in the clinical and scientific setting. "Precise" mainly covers technological precision in radiotherapy as well as targeted oncologic therapeutics. "Personalized" emphasizes biology-directed individualization of radiation treatment. Finally, "Human" underlines the patient-centered approach and points towards the need for individual longer-term career curricula for clinicians and researchers in the field.

Conclusion The vision 2030 balances the ambition of physical, technological, and biological innovation as well as a comprehensive, patient-centered, and collaborative approach towards radiotherapy \& radiation oncology in Germany.

Keywords Vision development $\cdot$ Radiation therapy $\cdot$ Image guidance $\cdot$ Precision oncology $\cdot$ Career development

During recent years, physical, technological, and clinical developments have led to improvements in radiation therapy precision, minimally invasive surgery, molecular targeted drugs, and immunotherapy. Radiation therapy plays an essential role in various multidisciplinary cancer treatment concepts in nearly all tumor entities in both curative and palliative intent. In order to further guide future development and strengthen the role of radiation oncology, the German Society for Radiation Oncology (DEGRO) working group of young clinicians and scientists (yDEGRO) and the DEGRO representation of associate and full professors (AKRO) initiated the development of a vision for the future of radiotherapy \& radiation oncology in Germany.

The process of vision development, its chronology, methodology, and the one-day strategy retreat held by the yDEGRO and AKRO is presented in Fig. 1. The final vision, "Innovative radiation oncology Together - Precise, Personalized, Human" (original German phrase Innovative Radioonkologie im Team - Präzise, Personalisiert, Menschlich) and the respective interpretation and program are summarized in Tables 1 and 2. Background material regarding the vision development process as well as supporting statements by the Working Group Radiation Oncology (ARO) of the German Cancer Society, the German Society of Medical Physics (DGMP), the German Society of Radiobiology Research (DeGBS), and the Professional Association of German Radiation Oncologists (BVDST) are given in the online supplement.

The interpretation of the term "Innovative radiation oncology" highlights the role of designing and initiating clinical trials and translational research, the development of individualized treatment concepts, and the identification of new indications for radiotherapy. The critical implementation steps are formation of a national strategy committee for radiation oncology research and the expansion and coordination of third-party funded research.

The term "Together" reflects the aspect of team building within radiation oncology professions, i.e., radiation oncologists, medical physicists, radiation biologists, radiation therapists, and nursing staff. Additionally, "Together" emphasizes interdisciplinary team building among radiation oncology professionals, medical oncologists, physicians from other medical disciplines, and associated nonmedical professionals, e.g., data scientists. The term also implies and emphasizes the promotion of young scientists and future scientific leaders. The central work program includes strengthening the multidisciplinary radiation oncology team and promoting closer collaborations both within and beyond radiation oncology departments, i.e., with cooperation partners. This considers a resident training rotation in cooperating disciplines, expanding clinician scientist programs, and establishing mentoring programs to inspire and encourage young researchers for a scientific career in translational research. Furthermore, the role of promoting inspiring medical, physical, and biological teaching is highlighted as a central mechanism to fascinate students for a career in radiation oncology.

The term "Precise" covers both the technical precision of image-guided adaptive radiation therapy and treatment with molecular targeted drugs within the context of multimodal oncologic treatments. The key work program involves expansion of imaging techniques and standardization of data acquisition and its use for approaches to artificial intelligence.

The term "Personalized" focuses on individualized treatment strategies that take into account biological, medical, and personal information and patients' needs. It also promotes the development of molecular, clinical, and imaging-based biomarkers for diagnosis, response evaluation, 


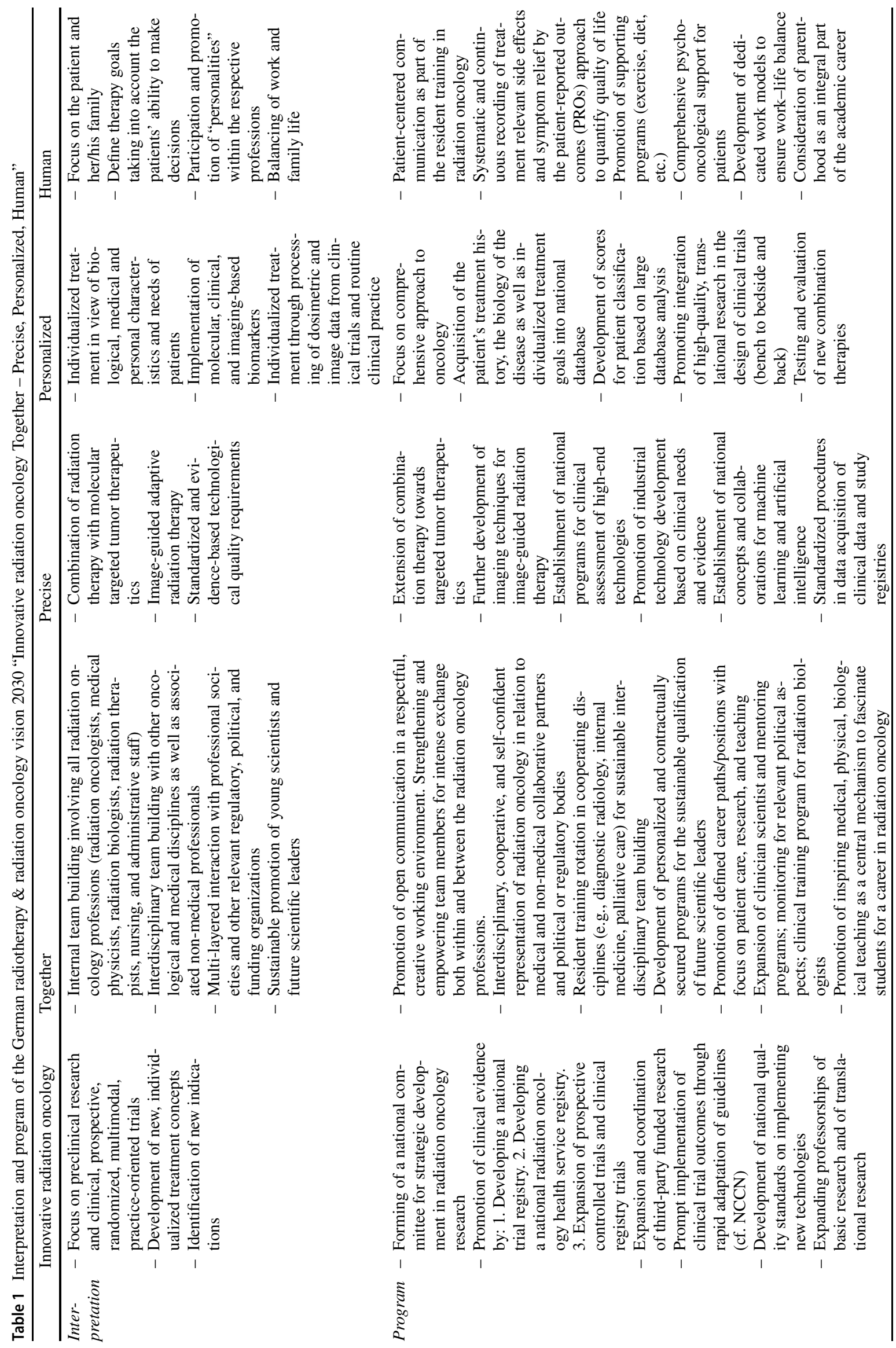




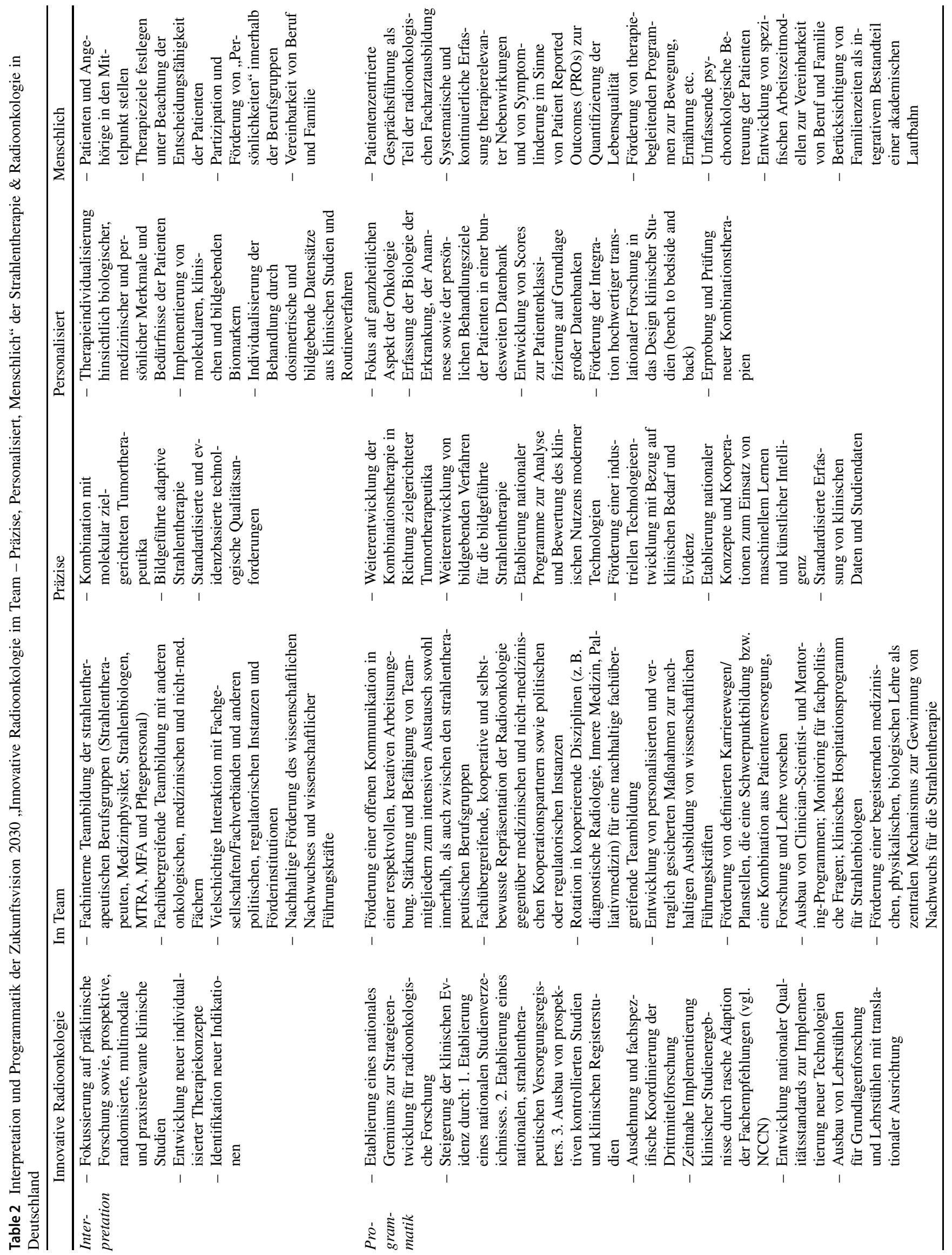




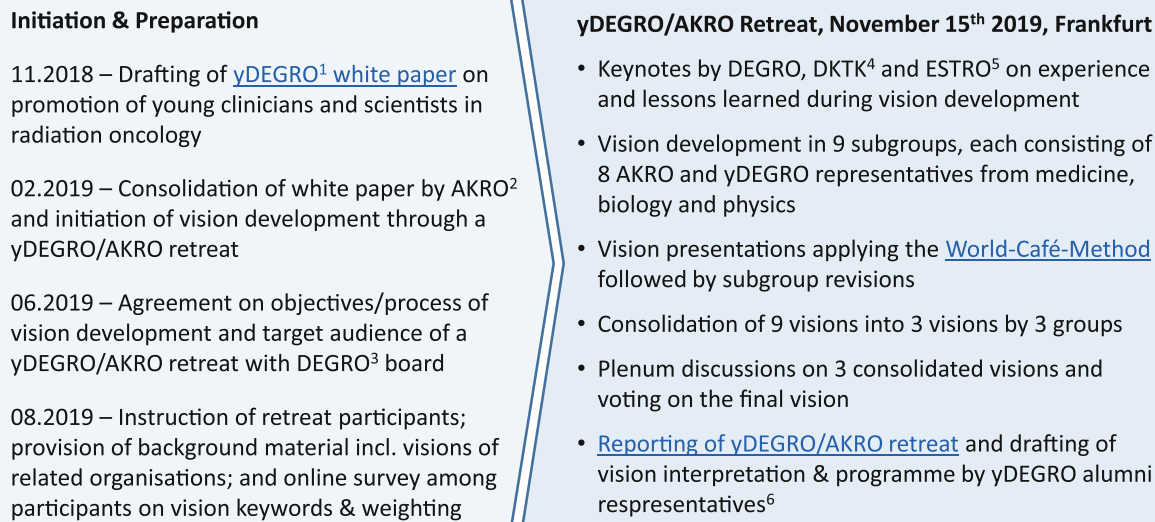

yDEGRO/AKRO Retreat, November $15^{\text {th }} 2019$, Frankfurt

- Keynotes by DEGRO, DKTK ${ }^{4}$ and ESTRO 5 on experience and lessons learned during vision development

- Vision development in 9 subgroups, each consisting of 8 AKRO and yDEGRO representatives from medicine, biology and physics

- Vision presentations applying the World-Café-Method followed by subgroup revisions

- Consolidation of 9 visions into 3 visions by 3 groups

- Plenum discussions on 3 consolidated visions and voting on the final vision

- Reporting of yDEGRO/AKRO retreat and drafting of vision interpretation \& programme by yDEGRO alumni respresentatives $^{6}$

Vision Interpretation \& Programme

08.2020 - Drafting of interpretation \& programme in 7 groups each consisting of 5 yDEGRO alumni representatives

11.2020 - Finalisation of draft interpretation \& programme by yDEGRO/AKRO retreat participants

12.2020 - Agreement on final interpret. \& programme with DEGRO board; commentary of associated boards of $\mathrm{BVDST}^{7}, \mathrm{DGMP}^{8}, \mathrm{DeGBS}^{9}, \mathrm{ARO}^{10}$

05.2021 - Dissemination of vision outcome; preparation of programme implementation by yDEGRO alumni

Fig. 1 Development process of German radiotherapy \& radiation oncology vision "Innovative radiation oncology Together - Precise, Personalized, Human." ${ }^{1}$ German Society for Radiation Oncology (DEGRO) working group of young clinicians and scientists and ${ }^{6}$ its alumni representatives as a link between young scientists and executives/leaders in radiotherapy and radiation oncology in Germany. ${ }^{2}$ Representation of associate and full professors of the DEGRO. ${ }^{3}$ German Society for Radiation Oncology. ${ }^{4}$ German Consortium for Translational Cancer Research. ${ }^{5}$ European Society for Radiotherapy and Oncology. ${ }^{7}$ Professional Association of German Radiation Oncologists. ${ }^{8}$ German Society of Medical Physics. ${ }^{9}$ German Society of Radiobiology Research. ${ }^{10}$ Working Group Radiation Oncology of German Cancer Society. This figure contains hyperlinks highlighted in blue, which are accessible through the corresponding figure version in the supplementary material. (https://link.springer.com/article/10.1007 \%2Fs00066-021-01843-9\#Sec1)

and prognosis. The key work program includes establishing a database for multicentric collection of clinical and biological data from routine practice and clinical trials. It also addresses the integration of high-quality translational research into the design of clinical trials (from bench to bedside and back). Data analysis and classification are based on methods of medical informatics.

The term "Human" underlines the need for a continuous patient-centered approach in radiation oncology. Innovation in radiation oncology serves to improve overall patient outcome and quality of life. Patients are empowered to make conscious, informed decisions about their treatment. Furthermore, this term emphasizes the promotion of "personalities" within the respective professions and acknowledges the need to promote flexible solutions and individual career paths to balance work and family life and open up long-term academic career pathways that cater to individual needs and strengths. The key work agenda contains the establishment of a resident training program for patient-centered communication and the facilitation of comprehensive programs for psycho-oncologic support, diet counselling, and physical exercise for cancer patients. Patient-reported outcomes (PROs) should be regularly measured in clinical trials and clinical routine.

In conclusion, the vision 2030 for radiotherapy \& radiation oncology in Germany reconciles the ambition of physical, technological, and biological innovation as well as a comprehensive, patient-centered, and cooperative approach in oncology.
Supplementary Information The online version of this article (https:// doi.org/10.1007/s00066-021-01843-9) contains supplementary material, which is available to authorized users.

Acknowledgements We would like to thank Claus Belka ("DEGROWhere Do We Come From?"), Stefan Joos ("Vision Development of the German Consortium for Translational Cancer Research") and Vincenzo Valentini ("Way to ESTRO Vision 2030 and Lessons Learned") for their introductory talks at the yDEGRO/AKRO strategy retreat. We thank Ilse Neuenhofen and Marcel Körrenz from "being to inspire" for their vital support regarding preparation and moderation of the yDEGRO/AKRO strategy retreat (for further information, see online supplement). Furthermore, we acknowledge the valuable input and ongoing commitment of all members of the yDEGRO alumni group as well as Nils Cordes, Ute Ganswindt, Hans Geinitz, and Andrea Wittig during preparation of this manuscript. We thank Verena Jendrossek (DeGBS), Mechthild Krause (ARO), Franz-Josef Prott (BVDST), Dimos Baltas, and Daniela Schmitt (both DGMP) for the supporting statements on behalf of their respective organization. Last but not least, we thank Rainer Fietkau for his valuable support as DEGRO president during the process of vision development.

Funding No specific funding was provided for this article.

Funding Open Access funding enabled and organized by Projekt DEAL.

Conflict of interest D. Krug has received honoraria from Merck Sharp \& Dome, outside of the submitted work. J. Hörner-Rieber received speaker fees and travel reimbursement from ViewRay Inc. as well as travel reimbursement from IntraOP Medical and Elekta Instrument $\mathrm{AB}$ and a grant from IntraOP Medical outside the submitted work. E. Sperk has received honoraria and travel support from Carl Zeiss Meditec AG, outside the submitted work. C. Seidel has received honoraria for lectures, consultation, or advisory board participation from the following for-profit companies: AbbVie, Bristol-Myers Squibb, HRA Pharma, medac, Roche, outside of the submitted work. C. Gani has received travel fees from Elekta. The Department of Radiation Oncology Tübingen receives financial and technical support from 
Elekta and Philips. C. Baues has received honoraria from Bristol Myers Squibb, outside of this submitted work. M. Hecht, N. Ebert, M. Mäurer, D.F. Fleischmann, E. Fokas, C. Straube, N.H. Nicolay, D. Schmitt, C. von Neubeck, C. Zamboglou, D. Kaul, J. Hess, S. Corradini, B. Frey, O. Blanck, T. Gauer, and M. Niyazi declare that they have no competing interests.

Open Access This article is licensed under a Creative Commons Attribution 4.0 International License, which permits use, sharing, adaptation, distribution and reproduction in any medium or format, as long as you give appropriate credit to the original author(s) and the source, provide a link to the Creative Commons licence, and indicate if changes were made. The images or other third party material in this article are included in the article's Creative Commons licence, unless indicated otherwise in a credit line to the material. If material is not included in the article's Creative Commons licence and your intended use is not permitted by statutory regulation or exceeds the permitted use, you will need to obtain permission directly from the copyright holder. To view a copy of this licence, visit http://creativecommons.org/licenses/by/4. $0 \%$.

\section{Affiliations}

\section{David Krug ${ }^{1}$ Markus Hecht ${ }^{2}$ - Nadja Ebert ${ }^{3}$ - Matthias Mäurer ${ }^{4}$ Daniel F. Fleischmann ${ }^{5}$ - Emmanouil Fokas ${ }^{6}$. Christoph Straube ${ }^{7,8}$. Nils Henrik Nicolay ${ }^{9}$ Juliane Hörner-Rieber ${ }^{10}$ - Daniela Schmittt ${ }^{11}$ Cläre von Neubeck ${ }^{12}$. Constantinos Zamboglou ${ }^{9}$ Elena Sperk ${ }^{13}$. David Kaul ${ }^{14}$. Julia Hess ${ }^{15}$. Stefanie Corradini ${ }^{5}$. Clemens Seidel ${ }^{16}$. Cihan Gani ${ }^{17}$. Christian Baues ${ }^{18}$ • Benjamin Frey ${ }^{2}$. Oliver Blanck ${ }^{1} \cdot$ Tobias Gauer $^{19}$ • Maximilian Niyazi ${ }^{5}$ (D)}

1 Department of Radiation Oncology, University Hospital Schleswig-Holstein, Kiel, Germany

2 Department of Radiation Oncology, Universitätsklinikum Erlangen, Friedrich-Alexander-Universität Erlangen-Nürnberg, Erlangen, Germany

3 Department of Radiotherapy and Radiation Oncology, Faculty of Medicine and University Hospital Carl Gustav Carus, Technische Universität, Dresden, Germany

4 Department of Radiotherapy and Radiation Oncology, University Hospital, Friedrich-Schiller-University, Jena, Germany

5 Department of Radiation Oncology, University Hospital, LMU Munich, Munich, Germany

6 Department of Radiotherapy and Oncology, University Hospital, Goethe University, Frankfurt, Germany

7 Department of Radiation Oncology, Klinikum rechts der Isar, Technical University of Munich, School of Medicine, Munich, Germany

8 RadioLog, Hof, Germany

9 Department of Radiation Oncology, Medical Center-University of Freiburg, Faculty of Medicine, University of Freiburg, Freiburg, Germany

10 Department of Radiation Oncology, Heidelberg University Hospital, Heidelberg, Germany
11 Department of Radiation Oncology, University Medical Center Göttingen, Göttingen, Germany

12 Department of Particle Therapy, University Hospital Essen, University of Duisburg-Essen, Essen, Germany

13 Department of Radiation Oncology, University Medical Center Mannheim, Medical Faculty Mannheim, Heidelberg University, Mannheim, Germany

14 Department of Radiation Oncology, Charité-Universitätsmedizin Berlin, Corporate Member of Freie Universität Berlin, Humboldt-Universität zu Berlin, Berlin, Germany

15 Research Unit Radiation Cytogenetics, Helmholtz Zentrum München, German Research Center for Environmental Health GmbH, Neuherberg, Germany

16 Klinik für Radioonkologie und Strahlentherapie, Universitätsklinikum Leipzig, Leipzig, Germany

17 Department of Radiation Oncology, Eberhard Karls Universität Tübingen, Tübingen, Germany

18 Department of Radiation Oncology and Cyberknife Center, University Hospital of Cologne, Cologne, Germany

19 Department of Radiotherapy and Oncology, University Medical Center Hamburg-Eppendorf, Hamburg, Germany 